\title{
CROSSED PRODUCTS AND MAXIMAL ORDERS
}

\author{
SUSAN WILLIAMSON
}

Introduction. Let $I$ be a maximal order over a complete discrete rank one valuation ring $R$ in a central simple algebra over the quotient field of $R$. The purpose of this paper is to determine necessary and sufficient conditions for $r$ to be equivalent to a crossed product over a tamely ramified extension of $R$.

It is a classical result that every central simple algebra over a field $k$ is equivalent to a crossed product over a Galois extension of $k$. Furthermore, it has been proved by Auslander and Goldman in [2] that every central separable algebra over a local ring is equivalent to a crossed product over an unramified extension.

Let $R$ denote a discrete rank one valuation ring. The set of maximal orders $M^{\prime}(R)$ over $R$ forms a subset of the set of hereditary orders $H^{\prime}(R)$ over $R$ (see [3]). An equivalence relation on the set of hereditary orders has been defined in [2]. Namely, if $A_{1}$ and $A_{2}$ are in $H^{\prime}(R)$, then $A_{1}$ is said to be equivalent to $\Lambda_{2}$ if there exist finitely generated free $R$-modules $E_{1}$ and $E_{2}$ and an $R$-algebra isomorphism

$$
\Lambda_{1} \otimes_{R} \operatorname{Hom}_{R}\left(E_{1}, E_{1}\right) \cong \Lambda_{2} \otimes_{R} \operatorname{Hom}_{R}\left(E_{2}, E_{2}\right) .
$$

It is established in [2] that an hereditary order which is equivalent to a maximal order is itself a maximal order.

The author has proved in [10] that every crossed product $\Delta(f, S, G)$ over a tamely ramified extension $S$ of a discrete rank one valuation ring $R$ is an hereditary order, and that $\Delta(f, S, G)$ is a maximal order if and only if the order of the conductor group $H_{f}$ is one (see Section 1 for the definition of $H_{f}$ ). She has also exhibited in this paper an example of a non-maximal hereditary order which is not equivalent to a crossed product over a tamely ramified extension. Now let $\Gamma$ be a maximal order over a complete discrete rank one valuation ring $R$ in a central simple algebra $\Sigma$ over the quotient field of $R$.

Received June 15, 1964.

Revised February 5, 1965. 
The main theorem of this paper states that a necessary and sufficient condition for $\Gamma$ to be equivalent to a crossed product over a tamely ramified extension of $R$ is the existence of a splitting field $K$ of $\Sigma$ for which

(1) the integral closure $S$ of $R$ in $K$ is a tamely ramified extension of $R$

(2) $f$ is in the image of the natural map $Z^{2}(G, U(S)) \rightarrow Z^{2}(G, U(K))$ where $f$ is a 2 -cocycle with the property that $\Delta(f, K, G)$ is equivalent to $\Sigma$.

At the end of the paper we present an example of a maximal order which is not equivalent to a crossed product over a tamely ramified extension.

The following notation shall be in use throughout the paper. If $R$ is a local ring, then $\bar{R}$ shall denote its residue class field. The multiplicative group of units of a ring $R$ shall be denoted by $U(R)$. Unless otherwise stated, $R$ shall always denote a complete discrete rank one valuation ring, $S$ the integral closure of $R$ in a finite Galois extension of the quotient field of $R$, and $G$ the Galois group of the quotient field extension. Since $R$ is complete, $S$ is also a complete discrete rank one valuation ring. The inertia group and the inertia ring of the extension $S$ of $R$ shall be denoted by $G_{l}$ ana $U$ respectively; and the image of a 2 -cocycle $f$ under the natural map $Z^{2}(G, U(S)) \rightarrow Z^{2}(G, U(\bar{S})$ ) shall be denoted by $\bar{f}$. For the definitions of crossed product, hereditary order, and tamely ramified extension we refer the reader to [10]. For convenience we recall that when the extension $S$ of $R$ is a tamely ramified extension of complete discrete rank one valuation rings then the inertia group $G_{I}$ is cyclic, and the $e^{t h}$ roots of unity are present in the inertia ring $U$, where $e$ is the order of $G_{l}$.

1. Cohomology and tame ramification. A crossed product over a tamely ramified extension is a maximal order if and only if its conductor group is trivial (see [10]). Therefore this section is devoted to the study of cohomology and the conductor group in the tamely ramified case.

Definition Let $S$ be a tamely ramified extension of a complete discrete rank one valuation ring $R$. For each cohomology class $[f]$ we define four subgroups of the cyclic group $G_{l}$ :

(1) $\Omega_{f}$ is the maximal subgroup of $G_{l}$ such that the image of $[f]$ under the restriction map $H^{2}(G, U(S)) \rightarrow H^{2}\left(\Omega_{f}, U(S)\right)$ is trivial,

(2) $I_{f}$ is the maximal subgroup of $G_{l}$ such that the image of $[\bar{f}]$ under the restriction map $H^{2}(G, U(\bar{S})) \rightarrow H^{2}\left(\Gamma_{f}, U(\bar{S})\right)$ is trivial, 
(3) $J_{f}$ is the maximal subgroup of $G_{l}$ with the property that [f] is in the image of the inflation map $H^{2}\left(G / J_{f}, U(S)\right) \rightarrow H^{2}(G, U(S))$,

(4.) $H_{f}$ is the maximal subgroup of $G_{l}$ with the property that $[\bar{f}]$ is in the image of the inflation map $H^{2}\left(G / H_{f}, U(\bar{S})\right) \rightarrow H^{2}(G, U(\bar{S}))$.

The group $H_{f}$ was named the conductor group in [10]. An element $f$ of $Z^{2}(G, U(S))$ is said to be properly normalized if $f$ is trivial on $\Omega_{f} \times \Omega_{f}$. Similarly, an element $\bar{f}$ in $Z^{2}(G \cdot U(\bar{S}))$ is said to be properly normalized if $\bar{f}$ is trivial on $\Gamma_{f} \times I_{f}$. The purpose of this section is to establish the equalities $\Omega_{f}=\Gamma_{f}$ and $J_{f}=H_{f}$.

Proposition 1.1. Let $S$ be a tamely ramified extension of a complete discrete rank one valuation ring $R$, and $f$ an element of $Z^{2}(G, U(S))$. Then $\Omega_{f}=\Gamma_{f}$, and $f$ is cohomologous to a properly normalized 2 -cocycle.

Proof. Since the image of $[f]$ under the restriction map $H^{2}(G, U(S)) \rightarrow$ $H^{2}\left(\Omega_{f}, U(S)\right)$ is trivial, certainly the image of $[\bar{f}]$ under the map $H^{2}(G, U(\bar{S}))$ $\rightarrow H^{2}\left(\Omega_{f}, U(\bar{S})\right)$ is trivial. Therefore $\Omega_{f} \subseteq \Gamma_{f}$.

Let $U$ denote the inertia ring of $S$ over $R$. Since $S$ is a tamely ramified extension of $R$ we know that $\bar{U}=\bar{S}$. To show that $\Gamma_{f} \subseteq \Omega_{f}$ we shall make use of the fact that $\Gamma_{f}$ is a cyclic group to first observe that the map $\Psi: H^{2}\left(\Gamma_{f}\right.$, $U(S)) \rightarrow H^{2}\left(\Gamma_{f}, U(\bar{U})\right)$ induced by the natural map $S \rightarrow \bar{S}=\bar{U}$ is a monomorphism. For let $\left[f_{\Gamma}\right]$ be in the kernel of $\Psi$, and let $u$ be an element of $U(T)$ such that $\left[f_{\mathrm{r}}\right]$ corresponds to $u \bmod N(U(S))$ under the canonical identification $H^{2}\left(\Gamma_{f}\right.$, $U(S))=U(T) / N(U(S))$ where $T$ is the integral closure of $R$ in the fixed field of $\Gamma_{f}$, and $N(U(S))$ denotes the norm of $U(S)$ in $T$. Since $\Psi\left(\left[f_{\mathrm{\Gamma}}\right]\right)$ is the identity we know that $(\bar{u})\left(\bar{c}^{n}\right)=\overline{1}$ for some element $\bar{c}$ in $U(\bar{U})$ where $n$ is the order of $I_{f}$ using the identification $H^{2}\left(\Gamma_{f}, U(\bar{U})\right)=U(\bar{U}) /(U(\bar{U}))^{n}$. Therefore the separable polynomial $\bar{P}(X)=X^{n}-1 / \bar{u}$ in $\bar{U}[X]$ has a root in $\bar{U}$. By Hensel's lemma it follows that $P(X)=X^{n}-1 / u$ has a solution, say $c$, in $U$. Therefore $N(c)=c^{n}$ and $u c^{n}=1$, and hence $f_{\Gamma}$ is cohomologus to the trivial 2cocycle and $\Psi$ is a monomorphism.

Now letting $f_{\Gamma}$ denote the restriction of $f$ to $\Gamma_{f} \times I_{f}$ it follows from the definition of $\Gamma_{f}$ together with the above observation that $f_{\Gamma}$ is cohomologous to the trivial 2 -cocycle in $Z^{2}\left(\Gamma_{f}, U(S)\right)$. Therefore there exists a map $\phi: I_{f}$ $\rightarrow U(S)$ such that $f_{\Gamma}(\sigma, \tau)=\phi(\sigma) \phi^{\top}(\tau) / \phi(\sigma \tau)$ for $\sigma$ and $\tau$ in $\Gamma_{f}$. Extend $\phi$ to 
$G$ by defining $\phi(\rho)=1$ if $\rho$ is not in $\Gamma_{f}$. Then the element $g$ of $Z^{2}(G, U(S))$ defined by $g(\sigma, \tau)=f(\sigma, \tau) \phi(\sigma \tau) / \phi(\sigma) \phi^{\sigma}(\tau)$ is cohomologous to $f$ and has the property that $g(\sigma, \tau)=1$ when $\sigma$ and $\tau$ are in $\Gamma_{f}$. Thus $\Gamma_{f} \cong \Omega_{f}$ and this concludes the proof.

In order to establish that $J_{f}=H_{f}$ we next prove three preliminary lemmas in which it is always assumed that $S$ is a tamely ramified extension of a complete discrete rank one valuation ring $R$.

LeMMA 1.2. For each element $f$ of $Z^{2}(G, U(S))$ there exists an element $g$ of $Z^{2}(G, U(S))$ such that $g$ is cohomologous to $f$, whenever $\rho$ is in $H_{f}$ it is true that $g(\tau, \rho)=1$, and $\bar{g}(\tau, \rho)=1$ if $\tau$ or $\rho$ is in $H_{f}$.

Proof. By Prop. 1.1 we may as well assume that $f$ is a properly normalized 2 -cocycle. Then $\vec{f}$ is also properly normalized. From the definition of $H_{f}$ we know by Prop. 2.3 of [10] that there exists a map $\bar{\phi}: G \rightarrow U(\bar{S})$ such that the 2-cocycle $\bar{g}$ in $H^{2}(G, U(\bar{S}))$ defined by $\bar{g}(\tau, \sigma)=\bar{f}(\tau, \sigma) \bar{\phi}(\tau) \bar{\phi}(\sigma) / \bar{\phi}(\tau \sigma)$ has the property that $\bar{g}(\tau, \sigma)=1$ if $\tau$ or $\sigma$ is in $H_{f}$, and that the restriction of $\bar{\phi}$ to $H_{f}$ takes values in the multiplicative group of $h^{\text {th }}$ roots of unity where $h$ is the order of $H_{f}$.

We shall use $\bar{g}$ to produce the definition of the desired 2-cocycle $g$. Let $G=\cup \tau_{j} H_{f}$ be a disjoint left coset decomposition of $G$ relative to the subgroup $H_{f}$, and let $\sigma$ now denote a generator of $H_{f}$. If $\bar{\phi}(\sigma)$ is the $h^{t h}$ root of unity $\bar{\eta}$, define $\phi(\sigma)$ to be $\eta$ where $\eta$ is an $h^{t, h}$ root of unity in $U(S)$ whose existence is guaranteed by the assumption that the extension $S$ of $R$ is a tamely ramified extension of complete local rings and Hensel's lemma.

For each $j$ define $\phi\left(\tau_{j}\right)$ and $\phi\left(\tau_{j \sigma}\right)$ by choosing representatives of $\bar{\phi}\left(\tau_{j}\right)$ and $\bar{\phi}\left(\tau_{j \sigma}\right)$ in $U(S)$ such that $g\left(\tau_{j}, \sigma\right)=1$ where $g\left(\tau_{j}, \sigma\right)$ is defined by $g\left(\tau_{j}, \sigma\right)=$ $f\left(\tau_{j}, \sigma\right) \phi(\tau j) \phi^{\tau}(\sigma) / \phi(\tau j \sigma)$. We next define $\phi\left(\tau j \sigma^{2}\right)$ to be a representative of $\bar{\phi}\left(\tau_{j} \sigma^{2}\right)$ for which $g\left(\tau_{j} \sigma, \sigma\right)=1$ where $g\left(\tau_{j} \sigma, \sigma\right)=f\left(\tau_{j} \sigma, \sigma\right) \phi\left(\tau_{j} \sigma\right) \phi^{\tau, \sigma}(\sigma) / \phi\left(\tau_{j} \sigma^{2}\right)$. Proceeding in this way we finally define $\phi\left(\tau_{j} \sigma^{n-1}\right)$ by choosing a representative of $\bar{\phi}\left(\tau j \sigma^{h-1}\right)$ for which $g\left(\tau ; \sigma^{h-2}, \sigma\right)=1$ where $g\left(\tau j \sigma^{h-2}, \sigma\right)=f\left(\tau j \sigma^{h-2}, \sigma\right) \phi\left(\tau j \sigma^{h-2}\right)$ $\phi^{\tau \sigma^{h-2}}(\sigma) ! \phi\left(\tau_{j} \sigma^{h-1}\right)$. Thus we have defined a map $\phi: G \rightarrow U(S)$. It remains to verify that the 2 -cocycle $g$ cohomologous to $f$ by $\phi$ satisfies the conclusion of the lemma. In order to do this we first check that the above definitions imply that $g\left(\tau ; \sigma^{n-1}, \sigma\right)=1$. Now 


$$
\begin{aligned}
g\left(\tau_{j} \sigma^{h-1}, \sigma\right) & =f\left(\tau_{j} \sigma^{h-1}, \sigma\right) \phi\left(\tau_{j} \sigma^{h-1}\right) \phi^{\tau_{j} \sigma^{h-1}}(\sigma) / \phi\left(\tau_{j}\right) \\
& =\prod_{i=0}^{h-1} f\left(\tau_{j} \sigma^{i}, \sigma\right) \phi^{\tau_{j} \sigma^{i}}(\sigma) / \prod_{i=0}^{h-2} g\left(\tau_{j} \sigma^{i}, \sigma\right) \\
& =f\left(\tau_{j}, \sigma^{h}\right)\left[\phi^{\tau_{j}}(\sigma)\right]^{h} \\
& =1
\end{aligned}
$$

since the $h^{\text {th }}$ root of unity $\phi(\sigma)$ is present in the inertia ring and hence is left fixed by $\sigma$.

Therefore $g(\tau, \sigma)=1$ for all $\tau$ in $G$ where $\sigma$ is a generator of $H_{f}$. We verify finally that $g\left(\tau, \sigma^{i}\right)=1$ for $1 \leq i \leq h$. From the associativity relation on $g$ together with the above, we have that $g\left(\tau, \sigma^{i}\right)=g\left(\tau \sigma^{i-1}, \sigma\right) g\left(\tau, \sigma^{i-1}\right) / g^{\tau}\left(\sigma^{i-1}, \sigma\right)$ $=1$ for $1 \leq i \leq h$ and therefore $g(\tau, \sigma)=1$ for all $\tau$ in $G$ and $\rho$ in $H_{f}$.

As in Prop. 2.1 of [10], for each element $\tau$ of $G$ we let $n(\tau)$ be the integer defined modulo $e$ by the relation $\tau \sigma \tau^{-1}=\sigma^{n(\tau)}$ where $\sigma$ is a generator of $G_{I}$ and $e$ is the order of $G_{l}$. With this definition it is easy to check that $\tau \rho \tau^{-1}=\rho^{n(\tau)}$ for each $\rho$ in $G_{l}$.

Lemma 1.3. Assume the notation of Lemma 1.2. Then there exists a 2. cocycle $\hat{g}$ in $Z^{2}(G, U(S))$ cohomologous to $g$ such that $\hat{g}(\tau, \rho)=\hat{g}\left(\rho^{n(\tau)}, \tau\right)=1$ for each element $\rho$ in $H_{\dot{f}}$ and $\tau$ in $G$.

Proof. Let $\rho$ be in $H_{f}$ and $\tau$ in $G$. Denote by $K$ the quotient field of $S$ and by $F$ the fixed field of $\left\{\rho^{n(\tau)}\right\}$. We first show that $N_{K / E}\left(g\left(\rho^{n(\tau)}, \tau\right)\right)=1$. By the assumption on $g$ and its associativity property we have that $g\left(\rho^{n(\tau)}, \tau \rho^{i}\right)$ $=g\left(\rho^{n(\tau)}, \tau\right)$ and $g\left(\rho^{i n(\tau)}, \tau\right)=g\left(\rho^{n(\tau)}, \tau \rho^{i-1}\right) g^{\rho^{n(\tau)}}\left(\rho^{(i-1) n(\tau)}, \tau\right)$ for all $i$. These equalities imply that $g\left(\rho^{j n(\tau)}, \tau\right)=\prod_{i=0}^{j-1} g^{g^{i n(\tau)}}\left(\rho^{n(\tau)}, \tau\right)$ for $1 \leq j \leq b$, from which it follows that $\prod_{i=0}^{b-1} g^{\rho^{i n(\tau)}}\left(\rho^{n(\tau)}, \tau\right)=g\left(\rho^{b n(\tau)}, \tau\right)=g(1, \tau)=1$ where $b$ is the order of $\left\{\rho^{n(\tau)}\right\}$. Thus $N_{K / F}\left(g\left(\rho^{n(\tau)}, \tau\right)\right)=1$.

Since $N_{K / F}\left(g\left(\rho^{n(\tau)}, \tau\right)\right)=1$ it follows from Th. 3 p. 171 of [11] and the fact that $K$ is a tamely ramified inertial extension of $F$ that $g\left(\rho^{n(\tau)}, \tau\right)=y^{\varphi^{n(\tau)}} \xi / y$ for some $y$ in $U(S)$ and $b^{t h}$ root of unity $\xi$. And $\xi=1$ since $\overline{g\left(\rho^{n(\tau)}, \tau\right)}=\overline{1}$. Now we may construct $\hat{g}$. Let $G=\cup H_{f} \tau_{j}$ be a disjoint coset decomposition of $G$ relative to $H_{f}$. Fix a generator $\sigma$ of $H_{f}$. For each $\tau$ in $G$ define $\phi(\tau)=1 / y$ where $\tau$ is in $H_{f} \tau_{j}$ and $y$ is an element of $U(S)$ for which $g\left(\sigma^{n\left(\tau_{j}\right)}, \tau_{j}\right)=y^{\jmath^{n\left(\tau_{j}\right)}} / y$. Now define $\hat{g}$ by $\hat{g}(\tau, \beta)=g(\tau, \beta) \phi(\tau) \phi^{\tau}(\beta) / \phi(\tau \beta)$. It is easy to verify that $\hat{g}$ has the desired properties. 
Lemma 1.4. Assume the notation of Lemma 1.3. Then there exists a 2cocycle $q$ in $Z^{2}(G, U(S))$ cohomologous to $\hat{g}$ and satisfying $q(\tau, \sigma)=1$ whenever $\tau$ or $\sigma$ is in $H_{f}$.

Proof. Let $G=\cup H_{f} \tau_{j}$ be a disjoint right coset decomposition of $G$ relative to the subgroup $H_{f}$. Define $\phi: G \rightarrow U(S)$ by $\phi\left(\sigma \tau_{j}\right)=1 / \hat{g}\left(\sigma, \tau_{j}\right)$ where $\sigma$ is in $H_{f}$. Define $q: G \times G \rightarrow U(S)$ by $q(\tau, \rho)=\hat{g}(\tau, \rho) \phi(\tau \sigma) / \phi(\tau) \phi^{\tau}(\rho)$. Let $\tau=\omega \tau_{j}$ be any element of $G$ where $\omega$ is in $H_{f}$, and let $\sigma$ be any element of $H_{f}$.

Then from the definition of $q$ we obtain the equality $q(\tau, \sigma)=q\left(\omega \tau_{j}, \sigma\right)=$ $\hat{g}\left(\omega \tau_{j}, \sigma\right) \hat{g}\left(\omega, \tau_{j}\right) / \hat{g}\left(\omega \sigma^{n\left(\tau_{j}\right)}, \tau_{j}\right)$. By the associativity relation satisfied by the 2-cocycle $\hat{g}$ we have that $\hat{g}\left(\omega \sigma^{n\left(\tau_{j}\right)}, \tau_{j}\right) \hat{g}\left(\omega, \sigma^{n\left(\tau_{j}\right)}\right)=\hat{g}\left(\omega, \sigma^{n\left(\tau_{j}\right)} \tau_{j}\right) \hat{g}^{\omega}\left(\sigma^{n\left(\tau_{j}\right)}, \tau_{j}\right)$; and together with the assumption on $\hat{g}$ this implies that $\hat{g}\left(\omega \sigma^{n\left(\tau_{j}\right)}, \tau_{j}\right)=\hat{g}(\omega$, $\left.\tau_{j} \sigma\right)$. Since $\hat{g}\left(\omega \tau_{j}, \sigma\right) \hat{g}\left(\omega, \tau_{j}\right)=\hat{g}\left(\omega, \tau_{j} \sigma\right) \hat{g}^{\prime \prime}\left(\tau_{j}, \sigma\right)=\hat{g}\left(\omega, \tau_{j} \sigma\right)$ we conclude that $g(\tau, \sigma)=1$.

On the other hand $q(\sigma, \tau)=q\left(\sigma, \omega \tau_{j}\right)=\hat{g}\left(\sigma, \omega \tau_{j}\right) \hat{g}^{\circ}\left(\omega, \tau_{j}\right) / \hat{g}\left(\sigma \omega, \tau_{j}\right)$. But $\hat{g}\left(\sigma, \omega \tau_{j}\right) \hat{g}^{\sigma}\left(\omega, \tau_{j}\right)=\hat{g}\left(\sigma \omega, \tau_{j}\right) \hat{g}(\sigma, \omega)=\hat{g}\left(\sigma \omega, \tau_{j}\right)$. Therefore $q(\sigma, \tau)=1$, and this concludes the proof.

Proposition 1.5. Let $S$ be a tamely ramified extension of a complete discrete rank one valuation ring $R$, and $f$ an element of $Z^{2}(G, U(S))$. Then $H_{f}=J_{f}$.

Proof. By the definition of $J_{f}$ there exists a 2-cocycle $g$ in $Z^{2}(G, U(S))$ such that $g$ is cohomologous to $f$ and $g(\sigma, \tau)=1$ if $\sigma$ or $\tau$ is in $J_{f}$. If $g$ is cohomologous to $f$ by $\phi: G \rightarrow U(S)$, then $\bar{g}$ is cohomologous to $\bar{f}$ by $\bar{\phi}: G \rightarrow$ $U(\bar{S})$. The fact that $g(\sigma, \tau)=1$ if $\sigma$ or $\tau$ is in $J_{f}$ implies that $J_{f} \subseteq H_{f}$.

To obtain the inclusion $H_{f} \subseteq J_{f}$ we apply the preceding lemmas to $f$, and so obtain a 2-cocycle $q$ in $Z^{2}(G, U(S))$ cohomologous to $f$ and satisfying $q(\sigma, \tau)$ $=1$ whenever $\sigma$ or $\tau$ is in $H_{f}$. It now follows from the definition of $J_{f}$ that $H_{f} \subseteq J_{f}$.

2. Maximal orders. In order to establish necessary and sufficient conditions for a maximal order to be equivalent to a crossed product over a tamely ramified extension in the complete case, the following lemma shall be useful.

Lемма 2.1. Let $\Sigma_{1}$ and $\Sigma_{2}$ be equivalent central simple $k$-algebras, where $k$ is the quotient field of a discrete rank one valuation ring $R$. If $\Gamma_{1}$ and $\Gamma_{2}$ are maximal orders in $\Sigma_{1}$ and $\Sigma_{2}$ respectively, then $\Gamma_{1}$ is equivalent to $\Gamma_{2}$. 
Proof. Since $\Sigma_{1}$ and $\Sigma_{2}$ are equivalent, there exist finitely generated $k$ modules $V_{1}$ and $V_{2}$ such that

$$
\Sigma_{1} \otimes_{k} \operatorname{Hom}_{k}\left(V_{1}, \quad V_{1}\right) \cong \Sigma_{2} \otimes_{k} \operatorname{Hom}_{k}\left(V_{2}, V_{2}\right) .
$$

Let $\Omega_{1}$ and $\Omega_{2}$ be maximal orders in $\operatorname{Hom}_{k}\left(V_{1}, V_{1}\right)$ and $\operatorname{Hom}_{k}\left(V_{2}, V_{2}\right)$ respectively. It is a classical result that $\Omega_{1}$ and $\Omega_{2}$ are of the form $\Omega_{1}=\operatorname{Hom}_{R}\left(E_{1}, E_{1}\right)$ and $\Omega_{2}=\operatorname{Hom}_{R}\left(E_{2}, E_{2}\right)$ where $E_{1}$ and $E_{2}$ are finitely generated free $R$-submodules of $V_{1}$ and $V_{2}$ respectively. Now $\Omega_{1}$ and $\Omega_{2}$ are central separable $R$-algebras, and therefore it follows from Prop. 8.6 of [2] that $\Gamma_{1} \otimes_{R} Q_{1}$ and $\Gamma_{2} \otimes_{R} \Omega_{2}$ are maximal orders. Since all maximal orders in a central simple algebra over a discrete rank one valuation ring are isomorphic (see Prop. 3.5 of [3]) we conclude that $\Gamma_{1} \otimes \Omega_{1} \cong \Gamma_{2} \otimes \Omega_{2}$. Therefore $\Gamma_{1}$ is equivalent to $\Gamma_{2}$.

Proposition 2.2. Let $S$ be a tamely ramified extension of a complete discrete rank one valuation ring $R$, and $f$ an element of $Z^{2}(G, U(S))$. Then every maximal order in the central simple k-algebra $\Delta(f, K, G)$ is equivalent to a crossed product over a tamely ramified extension of $R$.

Proof. By Lemma 1.4 we know that there exists a 2-cocycle $q$ in $Z^{2}(G$, $U(S))$ such that $q$ is cohomologous to $f$ and $q(\tau, \sigma)=1$ whenever $\tau$ or $\sigma$ is in $H_{f}$.

The subgroup $H_{f}$ is a normal subgroup of $G$, so that the fixed field $L$ of $H_{f}$ is a Galois extension of $k$ with Galois group $G / H_{f}$. Let $T$ denote the integral closure of $R$ in $L$ and observe that $T$ is a tamely ramified extension of $R$. To show that $q$ takes values in $U(T)$ we shall make use of the following definition. For each element $\tau$ of $G$ let $m(\tau)$ be the integer defined modulo $e$ by the relation $\tau^{-1} \omega \tau=\omega^{m(\tau)}$ where $\omega$ is a generator of the inertia group $G_{I}$ and $e$ is the order of $G_{l}$. We proceed to show that $q^{\circ}(\tau, \rho)=q(\tau, \rho)$ for all $\sigma$ in $H_{f}$ and all $\tau$ and $\rho$ in $G$. By the associativity property of $q$ we have the equalities

$$
\begin{aligned}
& q(\sigma, \tau \rho) q^{\tau}(\tau, \rho)=q(\sigma \tau, \rho) q(\sigma, \tau) \\
& q\left(\tau \sigma^{m(\tau)}, \rho\right) q\left(\tau, \sigma^{m(\tau)}\right)=q\left(\tau, \sigma^{m(\tau)} \rho\right) q^{\tau}\left(\sigma^{m(\tau)}, \rho\right)
\end{aligned}
$$

from which it follows that $q^{s}(\tau, \rho)=q(\sigma \tau, \rho)$ and also $q\left(\tau \sigma^{m(\tau)}, \rho\right)=q\left(\tau, \sigma^{m(\tau)} \rho\right)$. Therefore $q(\sigma \tau, \rho)=q\left(\tau \sigma^{m(\tau)}, \rho\right)=q\left(\tau, \rho \sigma^{m(\tau) m(\rho)}\right)$. And the equality

$$
q\left(\tau, \rho \sigma^{m(\tau) m(\rho)}\right) q^{\tau}\left(\rho, \sigma^{m(\tau) m(\rho)}\right)=q\left(\tau \rho, \sigma^{m(\tau) m(\rho)}\right) q(\tau, \rho)
$$


implies that $q^{\tau}(\tau, \rho)=q(\tau, \rho)$. Hence $q(\tau, \rho)$ is in the fixed field of $H_{f}$, and so $q$ takes values in $U(T)$.

We may now consider the crossed product $\Delta\left(g, T, G / H_{f}\right)$ where $g$ is the preimage of $q$ under the inflation map $Z^{2}\left(G / H_{f}, U(T)\right) \rightarrow Z^{2}(G, U(S))$. It follows from the definition of the conductor group $H_{f}$ and the second Noether isomorphism theorem, that the conductor group $H_{g}$ is trivial. Therefore we conclude from Theorem 2.5 of $[10]$ that $\Delta\left(g, T, G / H_{f}\right)$ is a maximal order in $\Delta\left(g, L, G / H_{f}\right)$. Now the central simple $k$-algebra $\Delta\left(g, L, G / H_{f}\right)$ is equivalent to $\Delta(q, K, G)$ (see [1]). If $\Gamma$ denotes a maximal order in $\Delta(q, K, G)$ it follows from the preceding lemma that $\Gamma$ is equivalent to the crossed product $\Delta\left(g, T, G / H_{f}\right)$.

Thus we have stablished the following main theorem.

THEOREM 2.3. Let $\Gamma$ be a maximal order over a complete discrete rank one valuation ring $R$ in a central simple algebra $\Sigma$. For $\Gamma$ to be equivalent to a crossed product over a tamely ramified extension of $R$ it is necessary and sufficient that there exists a splitting field $K$ of $\Sigma$ such that

(1) the integral closure $S$ of $R$ in $K$ is a tamely ramified extension of $R$

(2) $f$ is in the image of the natural map $Z^{2}(G, U(S)) \rightarrow Z^{2}(G, U(K))$ where $f$ is a 2-cocycle for which $\Delta(f, K, G)$ is equivalent to $\Sigma$.

Corollary 2.4. Let $\Sigma$ be a central simple algebra over the quotient field $k$ of a complete discrete rank one valuation ring $R$. If $\Sigma$ has a splitting field $K$ such that the integral closure $S$ of $R$ in $K$ is a tamely ramified inertial extension of $R$, then each maximal order $\Gamma$ in $\Sigma$ is equivalent to a crossed product over a tamely ramified extension of $R$.

Proof. We shall prove first that if an extension $S$ of $R$ is a tamely ramified inertial extension of complete discrete rank one valuation rings, then the natural map $H^{2}(G, U(S)) \rightarrow H^{2}(G, U(K))$ is an epimorphism, where $K$ denotes the quotient field of $S$, and $G$ is the Galois group of $K$ over $k$. Let $f$ be an element of $Z^{2}(G, U(K))$ and let $[f]$ correspond to $c \bmod N(U(K))$ under the canonical identification $\left.H^{2}(G, U(K))=U(k)\right) / N(U(K))$ which holds because $G$ is a cyclic group. As usual, $N$ denotes norm. Next write $c$ in the form $c=u p^{x}$ where $u$ is in $U(R), x$ is an integer, and $p$ denotes the prime element of $R$. Let $e$ be the order of $G$. Because of the assumption on $S$ and $R$ we know that for a 
proper choice of the prime element $P$ of $S$ it is true that $P^{e}=v p$ for some element $v$ in $U(R)$, and $\sigma(P)=\xi P$, where $\xi$ is a primitive $e^{t h}$ root of unity in $R$ and $\sigma$ is a generator of $G$ (see Prop. 3.1 of [10]). Therefore $N(P)= \pm v p$, and so the element $b=( \pm v p)^{-x}$ is also a norm. Hence $c b$ is an element of $U(R)$ which is congruent to $c \bmod N(U(K))$, and from this it follows that the map $H^{2}(G, U(S)) \rightarrow H^{2}(G, U(K))$ is an epimorphism.

Now we may prove the corollary. Since $\Sigma$ is split by $K$ we know that $\Sigma$ is equivalent to a crossed product $\Delta(f, K, G)$ for some element $[f]$ in $H^{2}(G$, $U(K)$ ), (see [1]). By the first part of the proof we may assume that $f$ is in the image of the natural map $Z^{2}(G, U(S)) \rightarrow Z^{2}(G, U(K))$. It now follows from the theorem that a maximal order $\Gamma$ in $\Sigma$ is equivalent to a crossed product over a tamely ramified extension of $R$.

Example 2.5. We present an example to show that a maximal order over a discrete rank one valuation ring need not be equivalent to a crossed product over a tamely ramified extension.

Consider the ring of polynomials $Z[X]$ with coefficients in the integers $Z$. Let $R=Z[X]_{(2)}$ be the localization of $Z[X]$ at the minimal prime ideal generated by the element 2. Let $K=k(\sqrt{2})$ where $k$ denotes the quotient field of $R$. Then the integral closure $S$ of $R$ in $K$ is $S=R[\sqrt{2}]$ and the Galois group $G$ of $K$ over $k$ is of order two. Note that $S$ is not a tamely ramified extension of $R$ since the field characteristic of $\bar{R}$ and the ramification index of $S$ over $R$ are both equal to two. Consider the element $[f]$ of $H^{2}(G, U(S))$ which corresponds to $X \bmod N(U(S))$ under the canonical identification $H^{2}(G, U(S))=$ $U(R) / N(U(S))$, and the crossed product $\Delta=\Delta(f, S, G)$.

It may be verified by computation that $\Delta \sqrt{ } 2$ is the unique maximal twosided ideal of $\Delta$. Since $\Delta \sqrt{2}$ is a free left $\Delta$-module it follows from Theorems 2.2 and 2.3 of [3] that $\Delta$ is a maximal order.

Suppose now that $\Delta(f, S, G)$ is equivalent to a crossed product $\Delta(g, T, H)$. We shall prove that $T$ cannot be a tamely ramified extension of $R$. The definition of equivalence implies that there exist finitely generated free $R$-modules $E_{1}$ and $E_{2}$ such that $\Delta(f, S, G) \otimes_{R} \operatorname{Hom}_{R}\left(E_{1}, E_{1}\right) \cong \Delta(g, T, H) \otimes_{R} \operatorname{Hom}_{R}\left(E_{2}, E_{2}\right)$. Let $\operatorname{rad} T=(A)$. Then the above isomorphism must map $\sqrt{ } 2$ into $A \imath$ where $u$ is a unit in $\Delta(g, T, H) \otimes \operatorname{Hom}\left(E_{2}, E_{2}\right)$. Therefore $A^{2}=2 v$ for some element $v$ in $U(T)$. Hence the ramification index of $T$ over $R$ is two, and so $T$ cannot 
be a tamely ramified extension of $R$.

\section{REFERENCES}

[1] E. Artin, C. Nesbitt and C. Thrall, Rings with Minimum Condition, Michigan, (1955).

[2] M. Auslander and O. Goldman, The Brauer group of a commutative ring, Trans. Amer. Math. Soc. Vol. 97 (1960), pp. 367-409.

[ 3 ] M. Auslander and O. Goldman, Maximal orders, Trans. Amer. Math. Soc. Vol. 97 (1960), pp. 1-24.

[4] M. Auslander and D. S. Rim, Ramification Index and Multiplicity, Ill. J. of Math. Vol. 7 (1963), pp. $566-581$.

[5] H. Cartan and S. Eilenberg, Homological Algebra, Princeton, (1956).

[6] M. Harada, Hereditary orders, Trans. Amer. Math. Soc. Vol. 107 (1963), pp. 273-290.

[7] D. G. Northcott, An Introduction to Homological Algebra, Cambridge University Press, (1960).

[8] O. Zariski and P. Samuel, Commutative Algebra, Vol. I, Van Nostrand, (1958).

[9] O. Zariski and P. Samuel, Commutative Algebra, Vol. II, Van Nostrand (1960).

[10] S. Williamson, Crossed Products and Hereditary Orders, Nagoya Math. J. Vol. 23 (1963), pp. $103-120$.

[11] N. Bourbaki, Elements de Mathèmatique, Livre II, Algèbre, Chap. V, Paris, Hermann, (1950).

\section{Harvard University \\ Cardinal Cushing College}

